\title{
MULTI-DIMENSIONAL FREE-ELECTRON LASER SIMULATION CODES: A COMPARISON STUDY*
}

\author{
$\underline{\text { S.G Biedron }}{ }^{1}$, Y.C. Chae, R.J. Dejus, B. Faatz ${ }^{2}$, H.P. Freund, ${ }^{3}$ \\ S.V. Milton, H-D. Nuhn ${ }^{4}$, S. Reiche ${ }^{2}$ \\ Advanced Photon Source, Argonne National Laboratory, Argonne, Illinois 60439 USA
}

\begin{abstract}
A self-amplified spontaneous emission (SASE) free-electron laser (FEL) is under construction at the Advanced Photon Source (APS). Five FEL simulation codes were used in the design phase: GENESIS, GINGER, MEDUSA, RON, and TDA3D. Initial comparisons between each of these independent formulations show good agreement for the parameters of the APS SASE FEL.
\end{abstract}

\section{INTRODUCTION}

The Advanced Photon Source (APS) at Argonne National Laboratory (ANL) is currently commissioning a free-electron laser (FEL) based on the self-amplified spontaneous emission (SASE) process [1]. The design parameters were based on capabilities of the existing APS linear accelerator, as well as on the results of various linear and nonlinear theoretical analyses. In this paper, we present a comparison of the results from linear theory [2], and five simulation codes. The codes used in the design include GENESIS [3], GINGER [4], MEDUSA [5], RON [6], and TDA3D [7,8]. Comparative simulations were performed for the parameters of the APS SASE FEL.

\section{CODE DESCRIPTIONS}

Before proceeding with the specific comparisons, a brief specification of the characteristics and capabilities of each of the simulation codes is necessary. Table 1 contains a brief listing of the more important properties of each of these codes. Of the five codes under consideration, only RON is limited to the linear regime; the other four are fully nonlinear. Three of the codes (GENESIS, GINGER, and MEDUSA) are fully polychromatic and can treat the full SASE spectrum. MEDUSA is also capable of treating an arbitrarily large spectrum of harmonics. All of the codes except MEDUSA use a wiggleraverage to treat particle dynamics; MEDUSA integrates the trajectories using the complete Lorentz force equations. The radiation field is obtained from a 3D (2D) field solver (FS) in TDA3D and GENESIS (GINGER) and from a 3D source-dependent expansion (SDE) in MEDUSA. Finally, all of the codes except GINGER are able to treat wiggler errors. Detailed descriptions of the codes are listed in the References section.

\footnotetext{
"Work supported by U.S. Department of Energy, Office of Basic Energy Sciences, under Contract Nos. W31-109-ENG-38 and DE-AC03-76SF00515.

${ }^{1}$ Email: biedron@aps.anl.gov

${ }^{2}$ Deutsches Elektronen Synchrotron, Notkestrasse 85, 22603 Hamburg, Germany.

${ }^{3}$ Permanent Address: Science Applications International Corporation, McLean, VA 22101, USA.

${ }^{4}$ Stanford Linear Accelerator Center, Stanford, California 94309, USA.
} 
Table 1: Code Properties

\begin{tabular}{|c|c|c|c|c|c|}
\hline Code & $\begin{array}{l}\text { Nonlinea } \\
\mathrm{r}\end{array}$ & $\begin{array}{l}\text { Poly- } \\
\text { chromatic }\end{array}$ & $\begin{array}{l}\text { Averaged } \\
\text { Orbits }\end{array}$ & Radiation Field & $\begin{array}{l}\text { Wiggler } \\
\text { Errors }\end{array}$ \\
\hline GENESIS & Yes & Yes & Yes & 3D FS & Yes \\
\hline GINGER & Yes & Yes & Yes & 2D FS & No \\
\hline MEDUSA & Yes & Yes & No & 3D SDE & Yes \\
\hline RON & No & No & Yes & 3D FS & Yes \\
\hline TDA3D & Yes & No & Yes & 3D FS & Yes \\
\hline
\end{tabular}

\section{CODE COMPARISONS}

The APS SASE FEL uses either a thermionic rf or photocathode of gun, the $650-\mathrm{MeV}, 2856-\mathrm{MHz}$ APS linac, two new transfer lines, and a new undulator hall with an optical diagnostics end station. The project will evolve over three phases, to reach saturation in the visible, UV, and VUV wavelength regimes, respectively. The design is based on known gun performance, constraints imposed by the APS linac, and the characteristics of currently available undulators. Tuning of the undulators has been optimized to meet the performance tolerances of the FEL. A set of parameters for the first phase was used for this comparison, and a Gaussian electron beam distribution was assumed. The specific parameters are summarized in Table 2 .

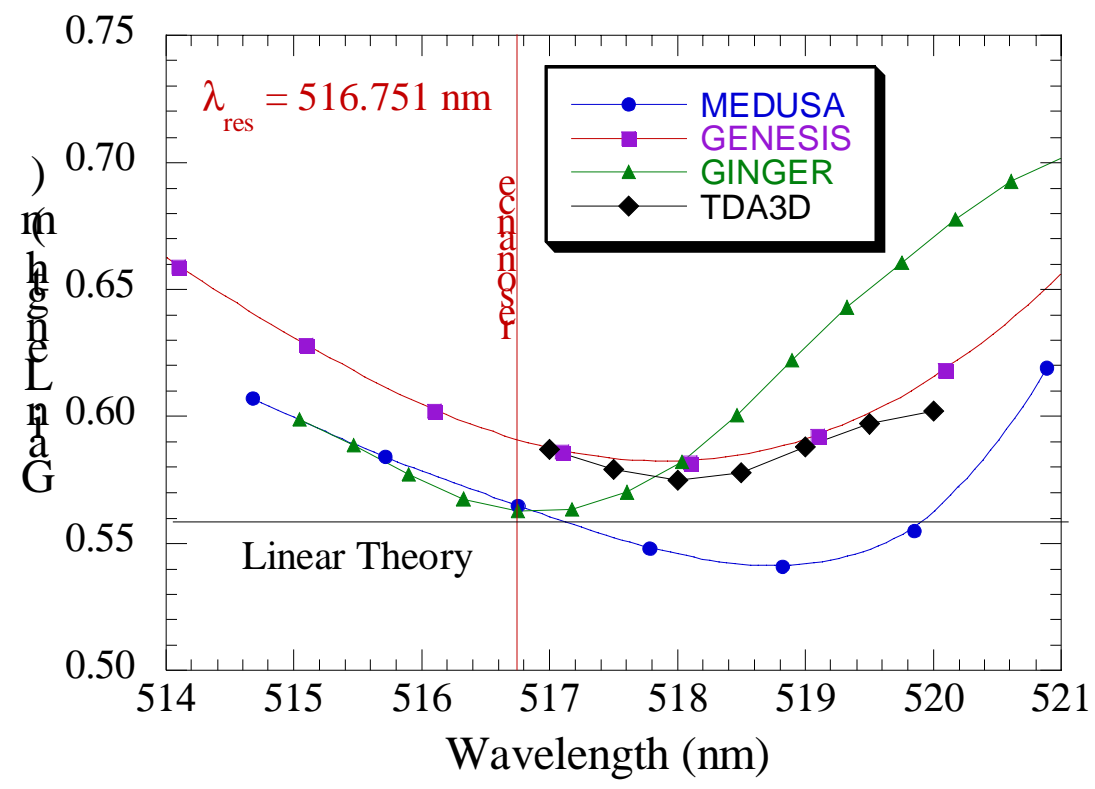

Fig. 1 Gain length versus wavelength for the nonlinear codes.

The optimum wavelength, corresponding to the minimum gain length, was obtained for each code by scanning in wavelength near the resonance. Note that the familiar 1D resonance formula $\left[\lambda=\lambda_{w}(1+\right.$ $\left.K^{2} / 2\right) / 2 \gamma^{2}$ ] yields a wavelength of $516.75 \mathrm{~nm}$. The results are shown in Fig. 1, where the gain length versus wavelength from MEDUSA, GENESIS, TDA3D, and GINGER are plotted. Such a scan is not available using RON. The optimal wavelengths for all five codes, however, are given in Table 3. Note that growth is not purely exponential in any of the nonlinear codes, and the gain length is dependent on the axial region chosen for the exponential fit. As a result, there is some uncertainty in the gain length and in the optimum wavelength, which impacts the saturated power. Nevertheless, the optimum wavelengths in GENESIS and TDA3D agree to within the accuracy of the procedure. The optimum wavelengths in RON and MEDUSA are also in close agreement and are slightly higher than in GENESIS and TDA3D (by $\sim 0.2 \%$ ). Note that in 
each of these codes, the optimum wavelength is slightly longer than the resonant wavelength. GINGER differs in that the optimum wavelength is very close to the $1 \mathrm{D}$ resonance.

Table 2: Simulation and Undulator Cell Parameters

\begin{tabular}{|c|c|}
\hline Parameter & Value \\
\hline$\gamma$ & 430.529 \\
\hline Normalized emittance & $5 \pi \mathrm{mm}-\mathrm{mrad}$ \\
\hline Peak current & $150 \mathrm{~A}$ \\
\hline Undulator period & $3.3 \mathrm{~cm}$ \\
\hline Undulator strength $(\mathrm{K})$ & 3.1 \\
\hline Energy spread & $0.1 \%$ \\
\hline Input start-up power & $1.0 \mathrm{~W}$ \\
\hline Undulator length & $2.4 \mathrm{~m}$ \\
\hline Focusing/diagnostics gap & $36 \mathrm{~cm}$ \\
\hline Quadrupole strength & $20 \mathrm{~m}^{-2}$ \\
\hline Quadrupole length & $5 \mathrm{~cm}$ \\
\hline
\end{tabular}

Table 3: Optimum Wavelengths

\begin{tabular}{lc}
\hline \hline Code & Optimum $\lambda(\mathrm{nm})$ \\
\hline \hline GENESIS & 517.78 \\
\cline { 1 - 1 } GINGER & 516.80 \\
\cline { 1 - 1 } MEDUSA & 518.82 \\
\cline { 1 - 1 } RON & 518.80 \\
\cline { 1 - 1 } TDA3D & 517.78 \\
\hline \hline
\end{tabular}

We consider a single-segment undulator with parabolic pole faces. The actual design uses multiple 2.763-m undulator "cells," each of which is composed of a 2.4-m magnetic segment and a 0.363-m section

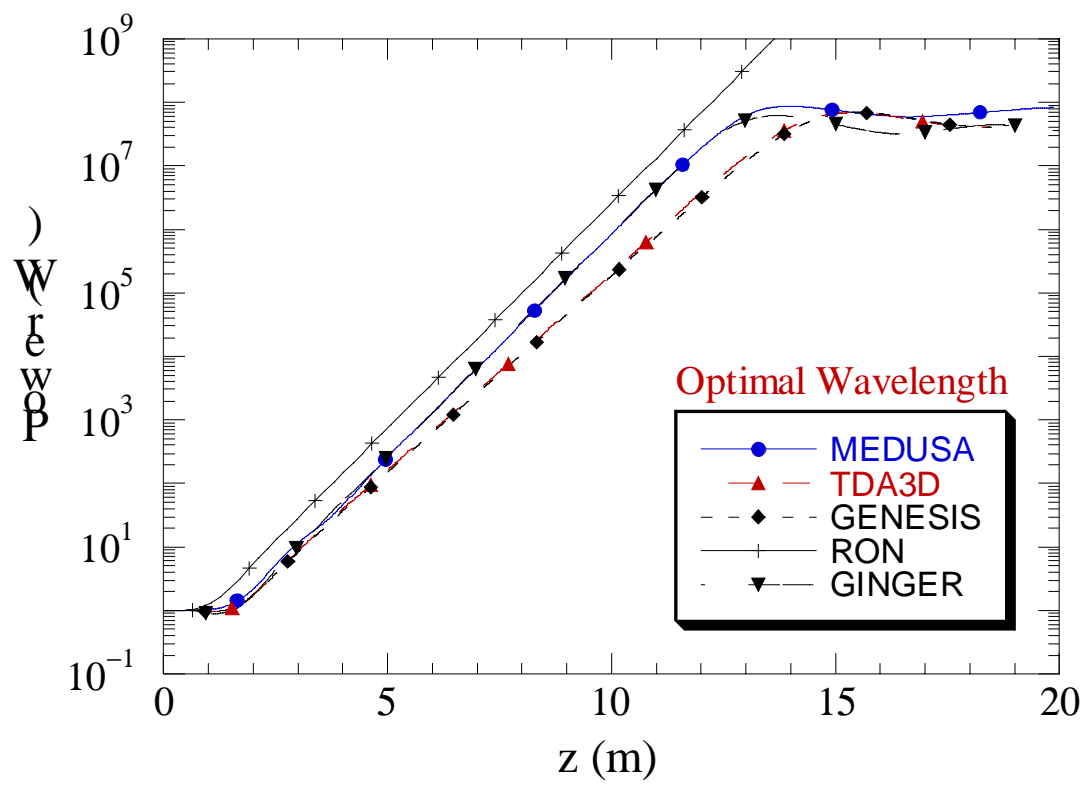

Fig. 2 Single segment case. 
for diagnostics, a combined quadrupole/corrector magnet, and drift space (see Table 2). The power versus distance along the undulator at the optimal wavelengths is shown in Fig. 2. The curves for GINGER and MEDUSA are almost identical and differ primarily in that GINGER predicts a somewhat lower saturated power. The calculated radiated power for RON is scaled from the bunched beam current density that is valid for the behavior in the exponential growth regime only where the radiated power is self-similar to the beam current. Thus, only the gain length in RON should be compared with the other codes. The gain length is almost identical in GINGER, MEDUSA, and RON. TDA3D and GENESIS yield nearly identical results, but the gain lengths are slightly longer than found with the other codes.

It should be noted that determining the gain length is not an unambiguous process in the nonlinear codes since these codes do not predict pure exponential growth (i.e., the derivative of the power versus distance is not precisely a constant along the length of the undulator). As a result, it is necessary to obtain a "best fit" to exponential growth that requires consideration of what length to choose; this introduces some

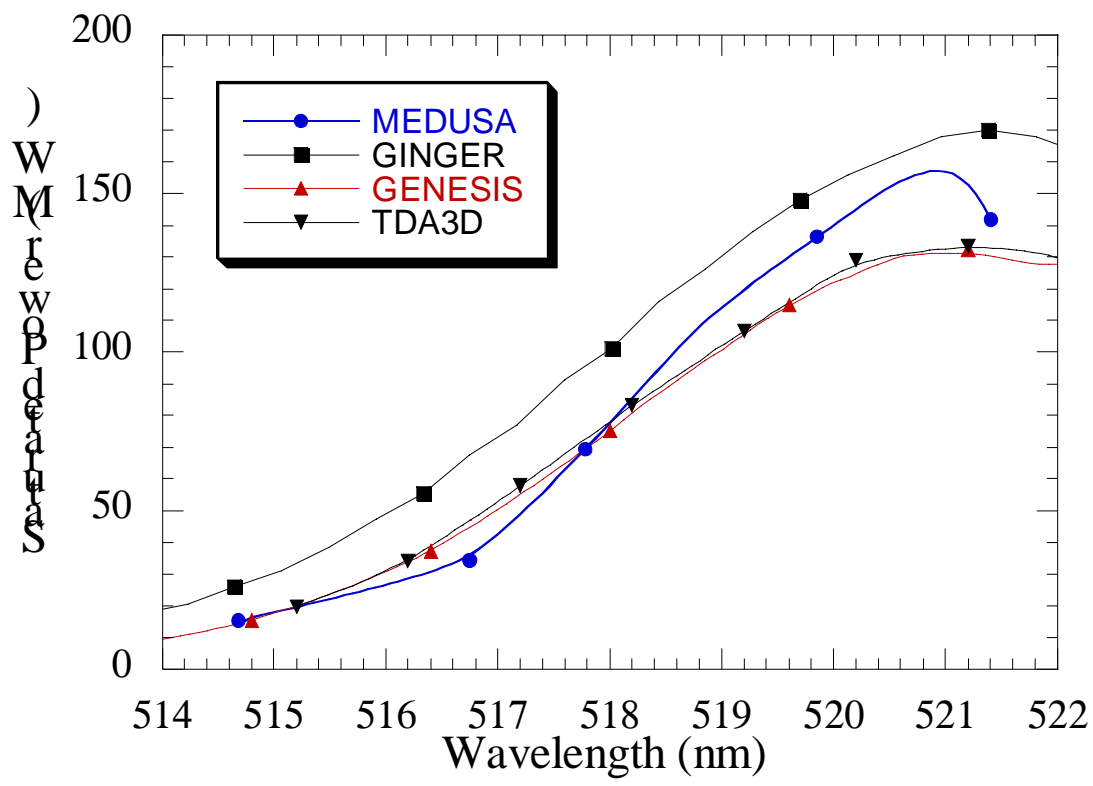

Fig. 3 Saturated power versus wavelength for the nonlinear codes.

uncertainty into the process. Differences of the order of $10-15 \%$ in the gain length from the different codes are within the range of uncertainty.

Some care should be used in interpreting the differences in the saturated power found between the codes at the optimum wavelength. The saturated power is a sensitive function of wavelength within the gain band, and small differences in the choices for the wavelength can result in relatively large variations in saturated power. This is illustrated in Fig. 3 where we plot the saturated power versus wavelength for the nonlinear codes. As shown in the figure, the nonlinear codes are in reasonable agreement, and the power increases from 10-20 MW at short wavelengths to between 120-170 MW at long wavelengths. Of the four codes, GINGER predicts the highest power at any given wavelength, while GENESIS and TDA3D predict the lowest. MEDUSA typically yields powers intermediate to those generated by the other three codes.

We now simulate the actual undulator design (less corrector fields) with flat pole face undulators and quadrupoles (Table 2) at the optimal wavelength found in the single-segment case. The power versus distance for the multi-segment case is shown in Fig. 4. Here, the shortest gain lengths are predicted by RON and GINGER, the longest by MEDUSA, while GENESIS and TDA3D predict intermediate gain lengths. In addition, the saturation powers in TDA3D and GENESIS are very close in this case, as are those predicted by GINGER and MEDUSA. 
Table 4 summarizes the saturation point and power for the single- and multiple-segment cases as determined by the nonlinear codes at the optimal wavelengths listed in Table 3 . Note that the saturated powers found in TDA3D and GENESIS, although not identical, are very close, while MEDUSA predicts somewhat higher and GINGER somewhat lower powers. It is not clear at this time why the saturated power predicted by GINGER and MEDUSA for the multi-segment case is so much higher than that found in TDA3D and GENESIS or why it is higher than the power found in the single-segment case. However, the saturated power is very sensitive to wavelength, and we speculate that there is some small retuning of the interaction for the multi-segment case due to differences in the beam dynamics.

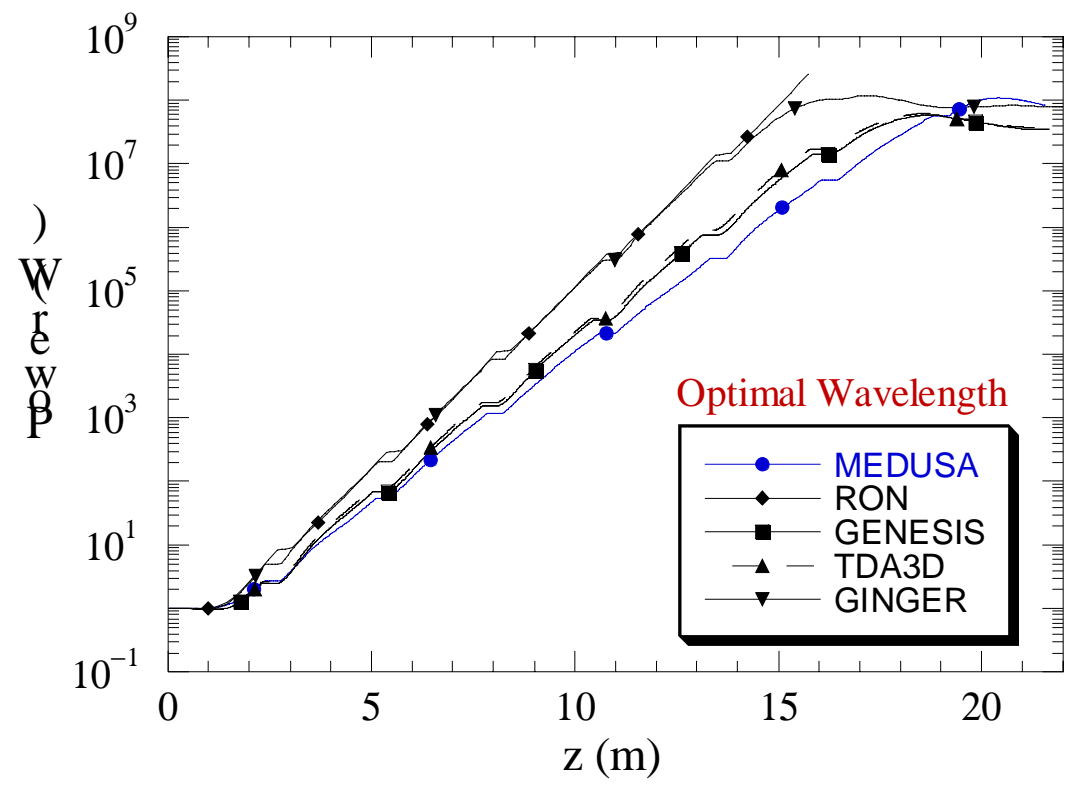

Fig. 4 Multiple segment case.

Table 4: Saturation Points and Power

\begin{tabular}{|c|c|c|c|c|}
\hline \multirow[t]{2}{*}{ Code } & \multicolumn{2}{|c|}{ Single-Segment } & \multicolumn{2}{|c|}{ Multi-Segment } \\
\hline & $L_{S A T}$ & $\mathrm{P}_{\mathrm{SAT}}$ & $\mathrm{L}_{\mathrm{SAT}}$ & $\mathrm{P}_{\mathrm{SAT}}$ \\
\hline "GENESIS & 15.5 & 69.4 MW & 18.8 & "58.0 MW \\
\hline GINGER & 13.7 & $61.7 \mathrm{MW}$ & 17.2 & $118 \mathrm{MW}$ \\
\hline MEDUSA & 14.0 & 87.4 MW & 20.8 & $109 \mathrm{MW}$ \\
\hline TDA3D & 15.4 & $68.9 \mathrm{MW}$ & 18.7 & $61.2 \mathrm{MW}$ \\
\hline
\end{tabular}

Wiggler imperfections have been simulated using TDA3D, MEDUSA, and GENESIS for the single-segment undulator. GINGER was omitted from this comparison because it cannot as yet treat wiggler imperfections, and RON was omitted because it was decided to study the effect of wiggler imperfections on the saturated power, and RON treats only the linear regime. In Figure 5, the efficiency found using the TDA3D, MEDUSA, and GENESIS is plotted versus the rms magnitude of the wiggler imperfections. A series of runs was made at a given $\left(\Delta B_{w} / B_{w}\right)_{r m s}$, each with a different error distribution. The number of runs used was determined by the requirement that the average efficiency converges to within $1 \%$ accuracy. The dots in the figure denote the ensemble average and the error bars denote the standard deviations about the average values. Of the three codes, GENESIS predicted the slowest decline in efficiency with increasing $\left(\Delta B_{w} / B_{w}\right)_{r m s}$, and TDA3D predicted the fastest. One reason why the decline is so rapid in TDA3D may be because it was run using the lowest order modes, which may not treat the required beam displacements with sufficient accuracy. However, the results from actual undulators constructed without careful sorting procedures can be expected to lie anywhere within the range indicated by the error 
bars. It is important to note that the ensemble averages found using the codes fall largely within the error bars; hence, substantial agreement is found in the description of wiggler imperfections.

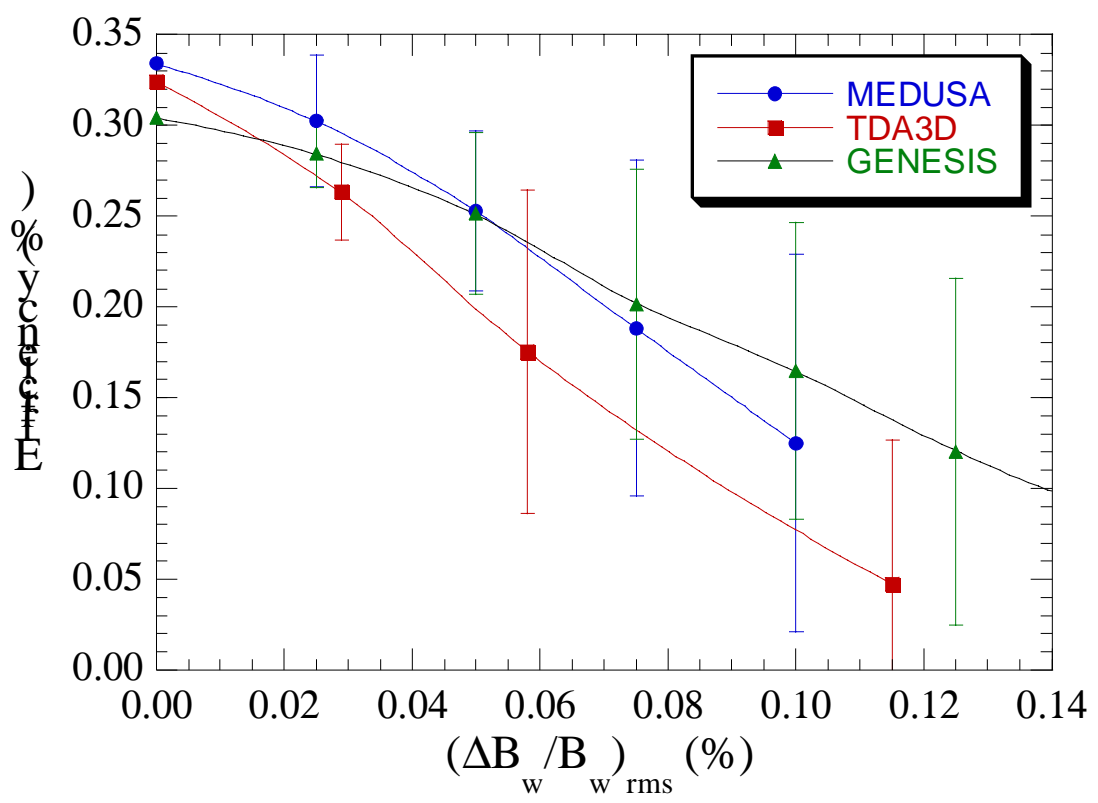

Fig. 5 Efficiency versus rms wiggler error.

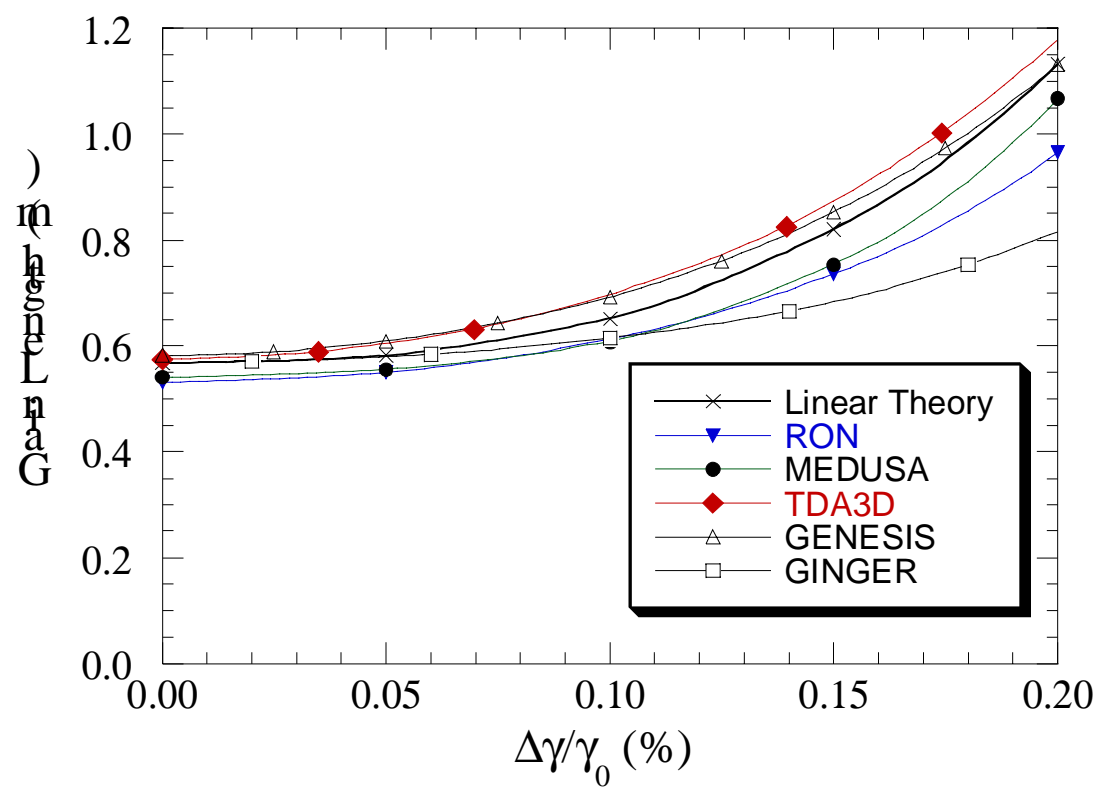

Fig. 6 Gain length versus energy spread. 


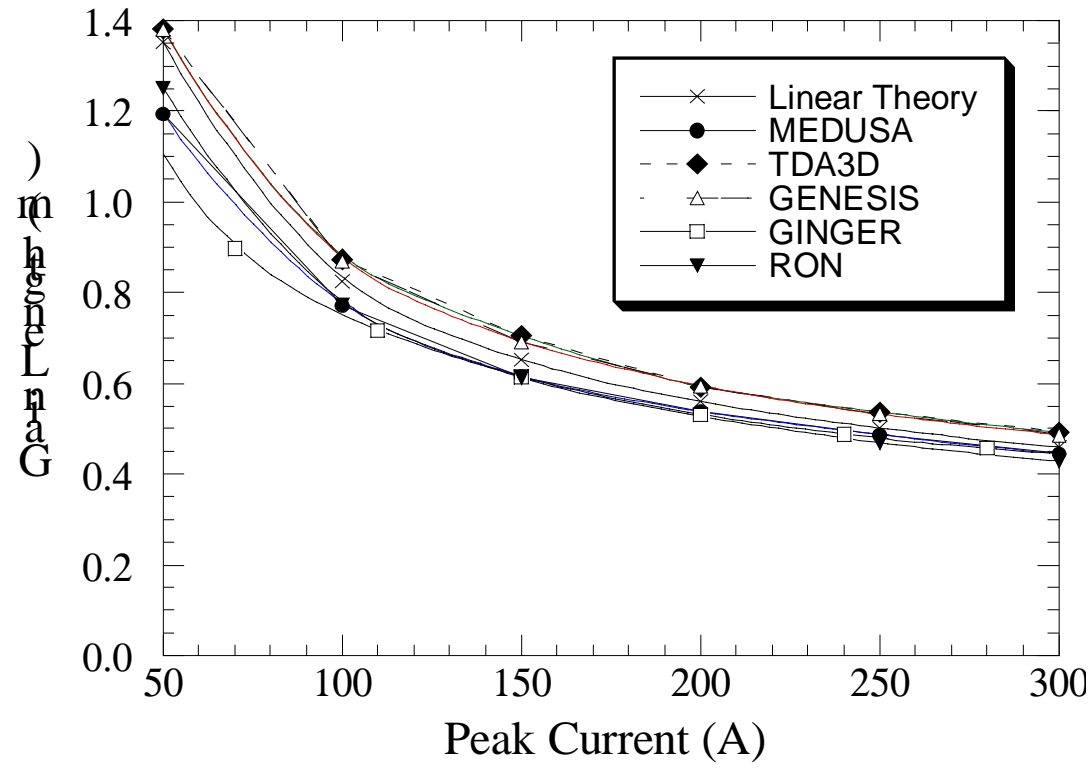

Fig. 7 Gain length versus peak current.

Comparisons of the gain length predicted by the codes and the linear theory for the single-segment case were also made. The energy spread was varied between $0.0-0.2 \%$, the peak current between $50-300 \mathrm{~A}$, and the normalized emittance between 1-10 $\pi \mathrm{mm}$-mrad. Figures 6,7 , and 8 show these variations. The codes are in reasonable agreement over the entire range of parameters studied. In general, it appears that GENESIS and TDA3D predict slightly longer gain lengths than the linear theory, while GINGER, MEDUSA, and RON predict slightly shorter gain lengths. Note that the linear theory is used for comparison purposes only, and should not be assumed as "perfect" but considered as an additional model. While the maximum discrepancies are of the order of $20 \%$ at some of the extremes of these parameter ranges, the maximum discrepancies are typically less than $15 \%$ for the parameters of interest in the APS SASE FEL. 


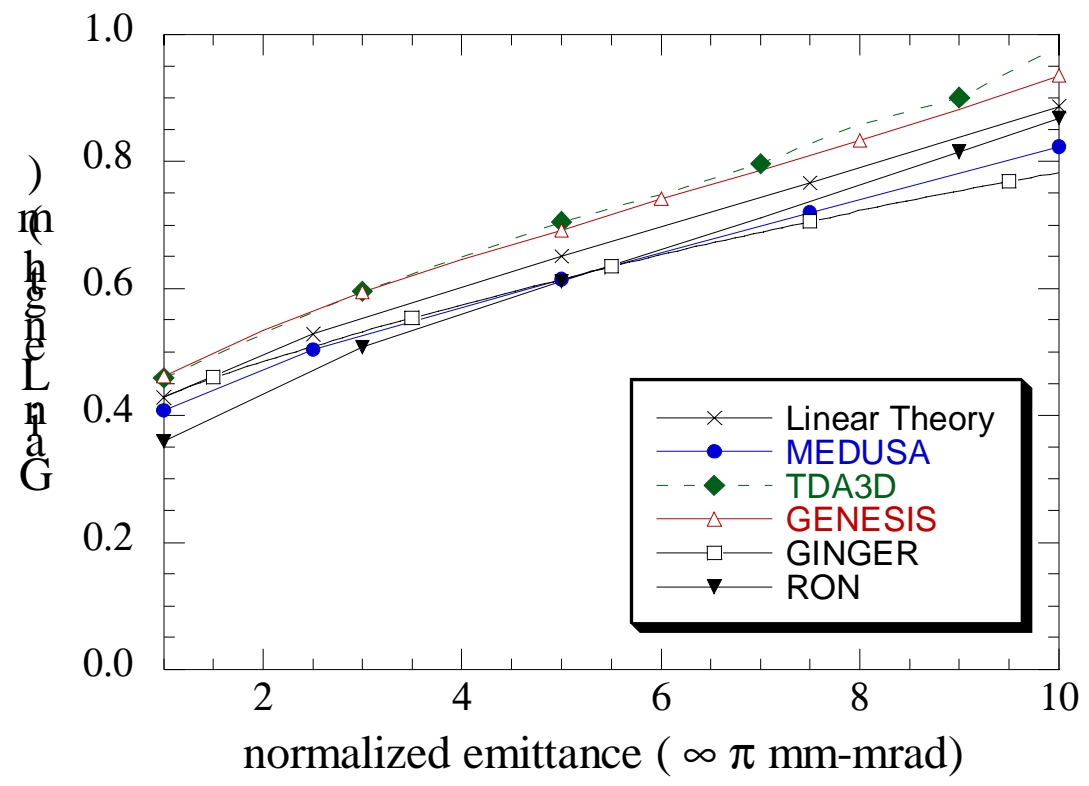

Fig. 8 Gain length versus normalized emittance.

\section{Conclusions}

In summary, GENESIS, GINGER, MEDUSA, RON, and TDA3D all show reasonable agreement with each other and with the linear theory for the first-phase APS SASE FEL parameters, giving greater confidence to the required length of undulator needed to reach full saturation.

\section{REFERENCES}

1. S.V. Milton et al., "FEL Development at the APS: The APS SASE FEL," in Free-Electron Laser Challenges II, Harold E. Bennett, David H. Dowell, Editors, Proceedings of SPIE, Vol. 3614, 86-95 (1999).

2. L.-H. Yu et al., Phys. Rev. Lett. 64, 3011 (1990); M. Xie, in Proceedings of the IEEE1995 Particle Accelerator Conference, 183 (1995).

3. S. Reiche, in NIM Proceedings of the 20th International FEL Conference (FEL98), Williamsburg, VA, USA, to be published.

4. W.M. Fawley, “An Informal Manual for GINGER and its post-processor XPLOTGIN,” LBID-2141, CBP Tech Note-104, UC-414 (1995).

5. H.P. Freund and T.M. Antonsen, Jr., Principles of Free-electron Lasers (Chapman \& Hall, London, 1986), 2nd edition; H.P. Freund, Phys. Rev. E, 52, 5401 (1995).

6. R.J. Dejus et al., in NIM Proceedings of the 20th International FEL Conference (FEL98), Williamsburg, VA, USA, to be published.

7. T.M. Tran and J.S. Wurtele, Computer Physics Communications 54, 263-272 (1989); S. Reiche and B. Faatz, in NIM Proceedings of the 20th International FEL Conference (FEL98), Williamsburg, VA, USA, to be published.

8. H.-D.Nuhn, "Overview of SASE Free-Electron Laser Simulation Codes," in Free-Electron Laser Challenges II, Harold E. Bennett, David H. Dowell, Editors Proceedings of SPIE, Vol. 3614, 119-130 (1999). 